\title{
A Selective Sulfide Oxidation catalyzed by Heterogeneous Artificial Metalloenzymes Iron@NikA.
}

\author{
Sarah Lopez, ${ }^{[\mathrm{a}],[\mathrm{b}]}$ Caroline Marchi-Delapierre ${ }^{[\mathrm{a}]}$ Christine Cavazza ${ }^{[\mathrm{a}]}$ and Stéphane Ménage ${ }^{\star[a]}$
}
[a] Dr S. Lopez, Dr C. Marchi-Delapierre, Dr C. Cavazza, Dr S. Ménage :
Univ. Grenoble Alpes, CEA, CNRS, IRIG, CBM, F-38000 Grenoble, France.
smenage@cea.fr ; http://www.cbm-lab.fr/en/Pages/BioCE/ArMs.aspx

[b] Dr S. Lopez

Univ. Grenoble-Alpes, DCM-SeRCO, Grenoble, France.

Supporting information for this article is given via a link at the end of the document.

\begin{abstract}
Performing a heterogeneous catalysis with proteins is still a challenge. Here, we demonstrate the importance of cross-linked crystals for sulfoxide oxidation by an artificial enzyme. The biohybrid consists of the insertion of an iron complex into a NikA protein crystal. The heterogeneous catalysts displays a better efficiency-with higher reaction kinetics, a better stability and expand the substrate scope compared to its solution counterpart. Designing crystalline artificial enzymes represents a good alternative to soluble or supported enzymes for the future of synthetic biology.
\end{abstract}

Introduction. Sustainable chemistry implies the discovery of new efficient but non-toxic solutions to synthesize molecules following eco-friendly strategies. ${ }^{[1]}$ Biocatalysis, boosted by the development of synthetic biology, represents a great opportunity to fulfill the requirements of green chemistry but still suffers from comparisons with the metal-based chemical catalysis. ${ }^{[2]}$ Biocatalysis and metalbased catalysis are two highly dynamic fields of research, ranging from enzyme and in cellulo processes to homogeneous or heterogeneous catalysis. Currently, each approach possesses advantages such as the mastering of chemo, regio and stereoselectivity and catalytic efficiency for enzymes while heterogeneous inorganic catalysts provide a better stability and recyclability. In order to optimize catalytic processes, enzymes can be engineered to gain stability and substrate promiscuity while MOFs, for example, are more and more sophisticated in order to allow the substrate control required to reach the expected selectivity. While natural enzymes have been engineered to gain higher activity ${ }^{[3]}$ or been repurposed for new reactivities, ${ }^{[4]}$ artificial enzymes issued of the seminal work of Whitesides et al., ${ }^{[5]}$ offer the opportunity to fill the gap between biocatalysis and chemical catalysis, providing new solutions for abiotic reactions with a special focus on stability. ${ }^{[6]}$

These hybrid systems are made of two building blocks, a protein chosen for its capacity to host tightly an unnatural active site, usually an inorganic catalyst. Both parts of these artificial enzymes have their own functionality, which is the control of the reaction selectivity for the protein and the nature of the reaction for the inorganic complex. This exciting strategy is eager to reproduce enzyme properties, in particular the kinetic and the enantioselectivity of a reaction. Stereoselectivity is nowadays well documented and sulfoxidation reaction is often a benchmark of hybrid systems as chiral sulfoxides centers are of great interest in the pharmaceutical industry, with the worldwide famous drugs Omeprazole ${ }^{\circledR}$ or Modafinil ${ }^{\circledR}{ }^{[7]}$ So far, only two systems have been engineered for API syntheses. ${ }^{\left[{ }^{[8]}\right.}$ Accordingly, the design of artificial sulfide oxgenases has been developed, differentiated by the mode of synthesis of the hybrid systems. First, the de novo active site approach consists of stabilizing the exogenous molecules (mostly Schiff bases complexes), by supramolecular interactions with the amino acids present inside a protein cavity (such as the Ni importer NikA, ${ }^{[9]}$ Xylanase $10 \mathrm{~A},{ }^{[10]}$ serum albumins ${ }^{[11]}$ or phytase). ${ }^{[12]}$ The Trojan horse strategy uses the same mode of interaction but with a natural substrate of the protein playing the role of an anchor. The latter is modified by a coordination moiety, as successfully demonstrated with streptavidin, ${ }^{[13}$ $\mathrm{BSA}^{[14]}$ or antibodies against steroids (testosterone) and the protein neocarzinostatin. ${ }^{[15]}$ Second, dative interactions between vanadate anions, iron or $\mathrm{Mn}$ and an amino acid is also demonstrated in the case of myoglobin ${ }^{[16]}$ or streptavidin. ${ }^{[17]}$ To ensure the stabilization of the non-natural active site in the cavity, a third approach uses covalent bonding via cysteine residues from engineered myoglobin or $\beta$ lactoglobulin as protein target. ${ }^{[18]}$ Finally, enzyme from scratch design is illustrated by the mimochrome series, in which the peptide environment is synthesized chemically and result of peptide self-assembly processes. ${ }^{[19]}$ All these systems provided from good to excellent selectivity for sulfoxide formation (up to 100\%) and stereoselectivity (up to $94 \%$ for serum albumins/Mn Schiff bases and streptavidin $/ \mathrm{VO}_{4}{ }^{2-}$ ) while yields oscillated between $50 \%$ and $100 \%$. The major difference between all these systems lies on the number of TON, a criterion for stability. A TON over 1000 was measured only in two cases, the mimochrome Mn Vlaa ${ }^{[19]}$ and FeLiBu/BSA. ${ }^{[14]}$ So far, industrial criteria for a use of artificial enzymes have not been reached and there is still a need for improvement. As observed in the field of chemical catalysis, the stability is increased when the molecular catalyst is grafted on surfaces and a similar approach can be used for enzymes. Several strategies exist from immobilization in/on MOFs or grafting on nanoparticles to encapsulation in a polymer matrix, micelle or liposome. ${ }^{[20]}$ One attractive strategy, i.e. the use of cross-linked enzyme crystals (CLEC), is quite astonishing because of its various assets. On the one hand, protein crystals achieve the highest enzyme concentration while preserving its integrity. In addition, the high solvent content affords the advantages of microporous materials, allowing the easy diffusion of molecules through solvent channels. On the other hand, with the CLEC technology, the intermolecular lattice contacts within the crystals and chemical cross-links formed with bifunctional reagents such as glutaraldehyde stabilizes enzymes. 
A

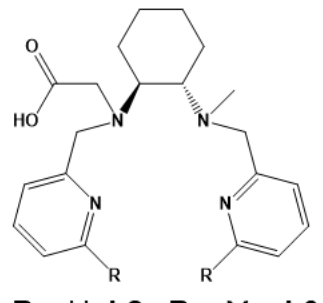

$\mathrm{R}=\mathrm{H}, \mathrm{L} 2 ; \mathrm{R}=\mathrm{Me}, \mathrm{L3}$

B NikA-FeL3 ${ }_{\text {cis } \beta}$

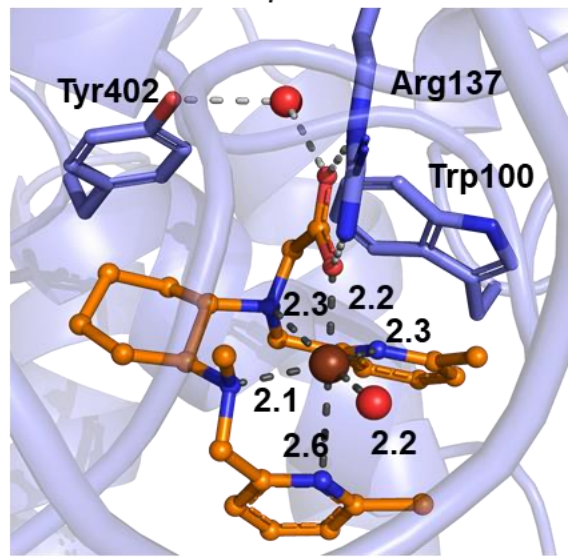

C NikA-FeL2 ${ }_{\text {trans }}$

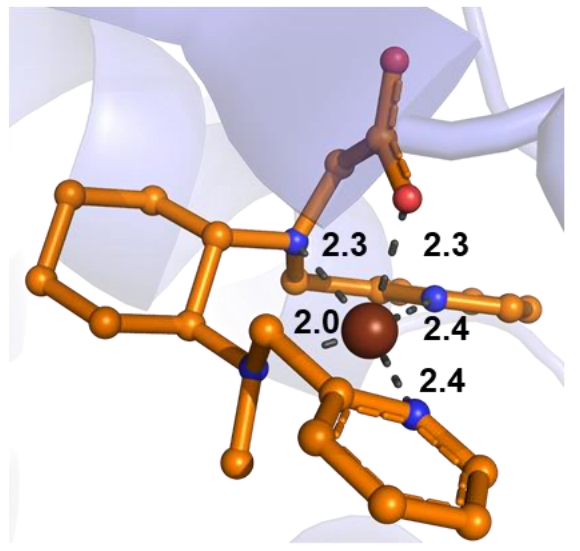

NikA-FeL2 ${ }_{\text {cis } \beta}$

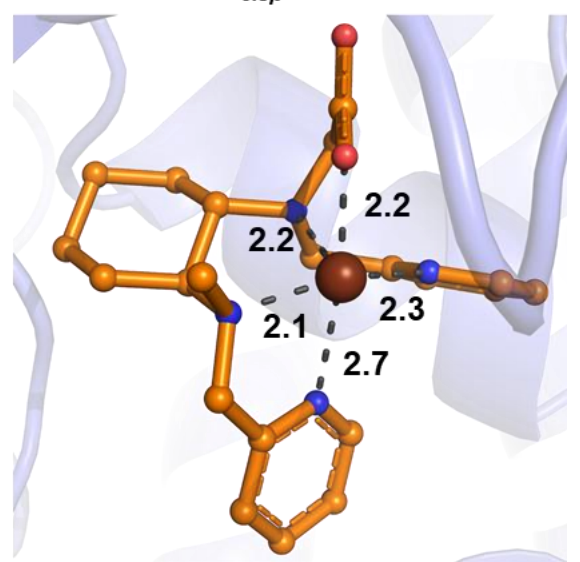

Figure 1. A: Structures of ligands L2 and L3. B: Crystal structure of NikA-FeL3 binding site. FeL3 under the only cis $\beta$ topology observed in this study. C: Crystal structure of NikA-FeL2 binding site. FeL2 under either the trans or the cisß topology, as observed in a previous study. ${ }^{[21]}$ Fe is depicted as a brown sphere. Distances are in $\AA$.

Remarkably, CLEC, as an immobilization technique, represents a great option to give access to biocatalysis in environments that are otherwise mostly incompatible with enzyme function, including prolonged exposure to high temperatures, extreme pH, near-anhydrous organic solvents and aqueous-organic solvent mixtures. ${ }^{[22]}$ Moreover, they can generally be stored indefinitely at room temperature. ${ }^{[23]}$ Up to now, the CLEC technology has been benchmarked as industrial biocatalysts for reductase or hydrolase activities but its application to redox metalloenzymes remains to be explored further. Notably, only Ueno et al. and our group have independently 
designed artificial metalloenzymes in cristallo for alkene transformations and $\mathrm{O}_{2}$ activation, showing the high impact of a crystalline hybrid catalyst on stability and activity. ${ }^{[24]}$

In order to benchmark this approach on thioether sulfoxidation, we designed cross-linked artificial sulfide oxidases in crystallo, based on our previous results obtained in solution, by the incorporation of iron complexes with nitrogen-based ligands into NikA. In this study, the results revealed the better catalytic efficiencies of heterogeneous CLECNikA-FeLn compared to their analogs in solution (so/NikAFeLn) on thioether oxidation, and particularly the stability of the CLECNikA-FeL3 system.

Results and discussion. Previously, we designed an artificial system that was capable of sulfoxidation in HEPES buffer solution. Thanks to a screening approach, sulfide molecules able to bind to the cavity of the nickel-binding protein NikA were selected. Among them, a series of substrates with the R1-S- $\mathrm{CH}_{2}-\mathrm{CONH}-\mathrm{R} 2$ motif $(S$ - and $\mathrm{N}$-substituted thioglycolamides, Scheme 1) have been transformed into their sulfoxides with high chemoselectivity, a TON of 199 and a TOF of 0.7 min $^{-1}$ for the substrate $\mathbf{1 b}$ in buffered solution. ${ }^{[9]}$ The reaction was catalyzed by the artificial enzyme so/NikA-FeL2, synthesized by the incorporation of an iron N2Py2 complex FeL2 into the NikA scaffold. L2 is a variation of the BPMCN ligand ${ }^{[25]}$ obtained by the replacement of one $N$-methyl moiety by a glycine moiety (Figure $1 \mathrm{~A}, \mathrm{R}=\mathrm{H}$ ). This modification ensures the stabilization of the complex FeL2 (with a Kd in the $\mu \mathrm{M}$ range) inside the NikA cavity via a salt bridge between the Arg137 and the carboxylate moiety. ${ }^{[21]}$ The X-ray structure of the hybrid revealed that the complex adopted different topologies, oscillating between a trans and a cis $\beta$ topology (differing by the pyridine ring positions to each other, Figure $1 \mathrm{C}$ ). In the trans topology (Figure 1C, top), the two pyridines are cis to each other in the equatorial plane of the complex while in the cis $\beta$ (Figure 1C, bottom), the two pyridine rings coordinate cis to each other but in perpendicular planes. This last topology has been observed in [6- $\left.\left.\mathrm{Me}_{2}-\mathrm{BPMCN}\right)(\mathrm{OTf})_{2}\right]$ (where OTf is trifluoromethanesulfonate anion). ${ }^{[25]}$ This mixture of topologies is deleterious to envisage an efficient metal based enantioselective control. This urged us to synthesize the dimethyl 6-Me ${ }_{2}-\mathrm{BPMCN}^{[25]}$ variant, FeL3 (Figure $1 \mathrm{~A}, \mathrm{R}=\mathrm{Me}$ ).

The ligand L3 and the complex syntheses are described in the supplementary material. It has to be noted that the ferrous complex appeared more appropriate to ensure its stabilization in the NikA cavity. Actually, compared to the ferric state, the ferrous state, with a charge of +1 should facilitate the insertion of the complex into the positively charged NikA binding site. Accordingly, the soaking was performed under an inert atmosphere. The complex FeL3 displayed a UV-visible spectrum characteristic of a ferrous ion featuring a unique charge transfer band in the visible region at $365 \mathrm{~nm}$, attributed to a transition from the Fe(II) "t2g" orbitals to the $\pi^{*}$ pyridine orbitals (Figure S1). ${ }^{[26]}$ The small absorption coefficient $\left(\varepsilon=750 \mathrm{~L} \cdot \mathrm{mol}^{-1} \cdot \mathrm{cm}^{-1}\right)$ indicates an iron high-spin state, $\mathrm{S}=2$. The complex was also identified by ESI-MS with a single positive fragment at $\mathrm{m} / \mathrm{z} 451$ (100\%) corresponding to a [L3-H+Fe(II)] $]^{+}$ion (Figure S2).

The X-ray structure of NikA-FeL3 was solved at $1.9 \AA$ resolution. Crystallographic data statistics are summarized in table S1. Two molecules are present in the asymmetric unit (called A and B). In both molecules, the ligand L3 is fully occupied while Fe(II) is only partly occupied (with an occupancy of about 0.7 ). For clarity, only molecule A will be discussed for the description of FeL3 binding mode in NikA cavity. In contrast to NikA-FeL2 (Figure 1C), the X-ray structure revealed that only one ligand conformation, the cis $\beta$ one, was observed (Figure 1B). The bulkiness of the methyl group on the pyridine rings has then led to the destabilization of the trans topology observed previously for NikA-FeL2. Depending on the structures, the Fe(II) ion is either pentacoordinated or hexacoordinated by two nitrogen atoms from the amino groups $(\mathrm{Fe}-\mathrm{N}=2.1$ and $2.3 \AA)$, one oxygen atom from the carboxylate group $(\mathrm{Fe}-\mathrm{O}=2.2 \AA)$ and one nitrogen atoms from one pyridine ring $(\mathrm{Fe}-\mathrm{N}=2.3 \AA)$. When $\mathrm{Fe}(\mathrm{II})$ is hexacoordinated, the nitrogen atom from the pyridine ring trans to the carboxylate group is bound to $\mathrm{Fe}(\mathrm{II})$ with a Fe-N distance of $2.6 \AA$. This pyridine group is flexible (with a B-factor of about 60 ) rendering the electron density map difficult to model and highlighting the lability of this coordination bond (Figure S3). Whatever the coordination number of the metal is, a water molecule binds to the iron $\left(\mathrm{Fe}-\mathrm{OH}_{2}=2.2 \AA\right)$. As previously observed in all structures of $\mathrm{NikA} /$ metal complexes hybrids described so far, FeL3 is mainly stabilized in the protein pocket by a salt bridge between the carboxylate group of L3 and Arg137. In addition, an interaction with a structural water molecule made one donating $\mathrm{H}$-bond with the oxygen atom of the carboxylate group involved in the salt bridge with Arg137, one accepting bond with the oxygen $\mathrm{OH}$ of Tyr402 and one donating $\mathrm{H}$-bond with the main chain $\mathrm{O}$ of Arg137. As in the case of NikA-FeL2 $2_{\text {cis } \beta}$ structure, one $\pi$-stacking interaction was also observed between one pyridyl group and the Trp100 side chain. Interestingly, in both structure, the ligand topology led to an elongation of the Fe- $\mathrm{N}_{\text {pyridine }}$ distance trans to the carboxylate, underlining a suspected trans effect of the anion. This effect is also combined with its position in an open space, excluding any interactions with the protein structure. 
<smiles>[R]NC(=O)CSc1cc([R])c([R])c([R2])c1[R7]</smiles>

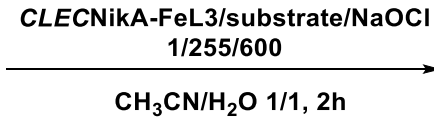

\begin{tabular}{c|cccccc} 
& $\mathbf{n}$ & $\mathbf{R 1}$ & $\mathbf{R}$ & $\mathbf{R}$ & $\mathbf{R} 4$ & $\mathbf{R} 5$ \\
\hline 1a & 0 & $\mathrm{H}$ & $\mathrm{H}$ & acetamide & $\mathrm{H}$ & $\mathrm{Ph}$ \\
1b & 0 & $\mathrm{H}$ & $\mathrm{H}$ & $\mathrm{Me}$ & $\mathrm{H}$ & $\mathrm{Ph}$ \\
1c & 0 & $\mathrm{H}$ & $\mathrm{OMe}$ & $\mathrm{OMe}$ & $\mathrm{H}$ & $\mathrm{Ph}$ \\
1d & 0 & $\mathrm{OMe}$ & $\mathrm{H}$ & $\mathrm{H}$ & $\mathrm{OMe}$ & $\mathrm{Ph}$ \\
1e & 1 & $\mathrm{H}$ & $\mathrm{H}$ & $\mathrm{H}$ & $\mathrm{H}$ & $\mathrm{Ph}$
\end{tabular}

Scheme 1. Details of the sulfoxidation reaction and the substrates used in this study.<smiles>[R]CNC(=O)CS(=O)(=O)C(Cl)(Cl)C(=O)N[R5]</smiles>

\begin{tabular}{c|cccccc} 
& $\mathbf{n}$ & $\mathbf{R 1}$ & $\mathbf{R}$ & $\mathbf{R}$ & $\mathbf{R}$ & $\mathbf{R}$ \\
\hline $\mathbf{1 f}$ & 0 & $\mathrm{H}$ & $\mathrm{H}$ & acetamide & $\mathrm{H}$ & Naphthyl \\
$\mathbf{1 g}$ & $\mathrm{O}$ & $\mathrm{H}$ & $\mathrm{H}$ & $\mathrm{Me}$ & $\mathrm{H}$ & Naphthyl \\
$\mathbf{1 h}$ & 0 & $\mathrm{OMe}$ & $\mathrm{H}$ & $\mathrm{H}$ & OMe & Naphthyl \\
$\mathbf{1 i}$ & 1 & $\mathrm{H}$ & $\mathrm{H}$ & $\mathrm{H}$ & $\mathrm{H}$ & Naphthyl
\end{tabular}

Confident that the complex was stabilized in NikA crystals, the CLEC synthesis was then undertaken in two steps. First, NikA-FeEDTA crystals were soaked with either FeL3 or FeL2 for four days in a glove box. The crystals were then soaked with a solution of glutaraldehyde for 5 hours to yield CLECNikA-FeL2 and CLECNikA-FeL3, which were subsequently washed and stored in a 1:1 solution of $\mathrm{CH}_{3} \mathrm{CN}: \mathrm{H}_{2} \mathrm{O}$.

To ascertain the role of the crystalline material on the catalysis, we have first undertaken to use the substrates of our earlier study in $2013^{[9]}$ under the similar reaction conditions but the solvent, here a mixture of aerated water and acetonitrile (Scheme 1). This medium will then help to dissolve water-insoluble substrates, in order to evaluate the substrate scope of the biohybrid catalysts. Actually, CLECNikA-FeL2 and CLECNikA-FeL3 (100 CLEC, $31 \mu \mathrm{M})$ catalyzed the oxidation by $\mathrm{NaOCl}$ of a series of nine thioglycolamides 1ai. CLECNikA-FeLn were compared to the complexes alone and so/NikA-FeL2 for 1a oxidation. ${ }^{\left[{ }^{9]}\right.}$ For each biohybrid, protein concentration was measured either by Size Exclusion Chromatography coupled to Multi-Angle-Laser-Light-Scattering (SEC-MALLS) or by using the Rose Bengal protocol. ${ }^{[27]}$ The metal content was measured by ICP-AES and the metal/protein ratio was closed to one for all biohybrids. The determination of X-ray structures of the different NikA/complexes hybrids demonstrated the full occupancy of FeLn inside NikA molecules (see supplementary material).

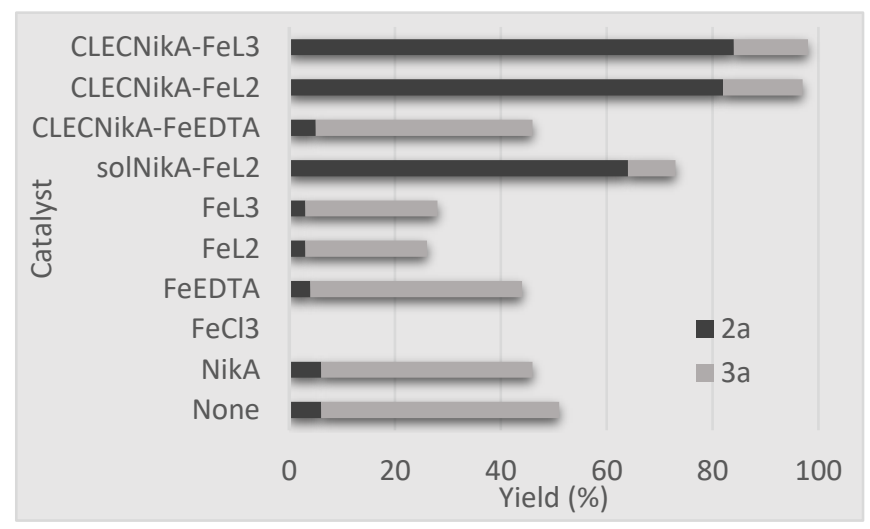

Figure 2. 1a oxidation by various catalysts under the following conditions: Catalyst:NaOCl:1a : 1:255:600; [cat] = $37 \mu \mathrm{M}$ in HEPES 10 mM pH 7; 2 hours except for CLEC [cat] $=31 \mu \mathrm{M}$ in $\mathrm{CH}_{3} \mathrm{CN}: \mathrm{H}_{2} \mathrm{O} 1 / 1$ v:v ; 2 hours

First, the uncatalyzed reaction led to a small amount of sulfoxide (less than $5 \%$ ) and an achiral formation of the dichlorinated product $3 \mathbf{a}$ (up to $5 \%$ ) (Scheme 1). A similar lack of reactivity was observed with any iron salts or the FeLn complexes alone. It has to be noted that the protein NikA itself is inactive, while other biomolecules such as phytase or serum albumin produced sulfoxides. ${ }^{[11 \mathrm{~b}, 28]}$ Conversely, the so/NikA-FeL2 hybrid, in buffered solution, produced sulfoxide 2a with a moderate yield and high selectivity (64\% (163 TON) and $90 \%$, respectively) (Table S2). The protein host helps to greatly annihilating the production of $\mathbf{3 a}$, causes a drastic gain in selectivity and a twenty-fold increase in yield. Second, oxidation of $\mathbf{1 b}$ was performed with so/NikA-FeL2 and so/NikA-FeL3. 2b was formed with a slight difference in yields, $63 \%$ versus $50 \%$, respectively. Third, oxidation of 1a was performed with CLECNikA-FeL2 and CLECNikA-FeL3. Using comparable catalyst concentrations, the yield in sulfoxide 2 a was increased by nearly $30 \%$ ( $82 \%$ (209 TON) and $84 \%$ (214 TON), for CLECNikA-FeL2 and CLECNikA-FeL3, respectively) and the selectivity was kept (85 vs $90 \%)$. Higher efficiencies were then obtained when switching to CLEC with the reference substrate. It is also proven that the complex FeLn controls the reactivity, as the CLECNikA-FeEDTA was fully inactive. The similar results obtained for both CLECNikA-FeL2 and CLECNikA-FeL3 indicate that the methyl substitution on the pyridine ring were not deleterious for the reactivity. However, the presence of only one ligand topology for CLECNikA-FeL3 has no effect at all on the enantioselectivity of the sulfoxidation since an enantioselective excess of only $10 \%$ was observed whatever the complex is, as in solution. 


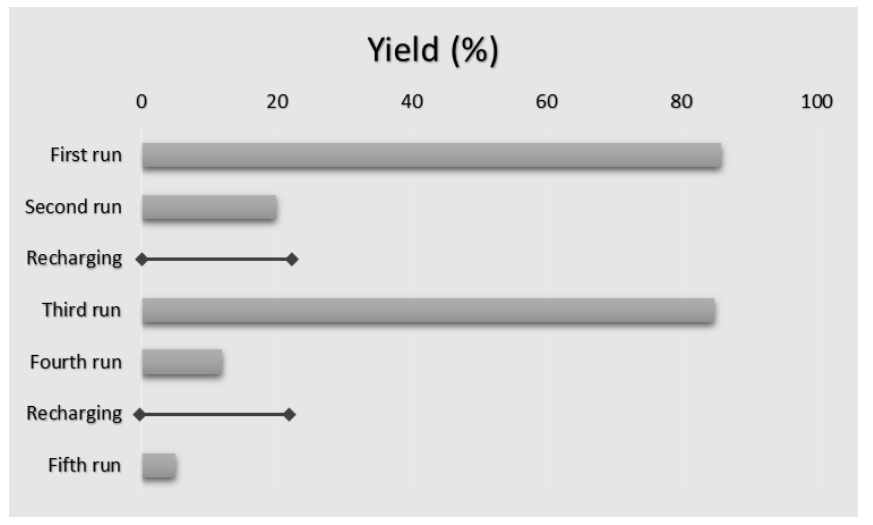

Figure 3. Yields in 2a during recharging experiment with CLECNikA-FeL3 as catalyst and 1a as substrate in standard experimental conditions (100 CLEC).

The CLECNikA-FeL3 stability was also evaluated (Figure 3). The 1a transformation was tested with a batch of $100 \mathrm{CLEC}$. At the end of the first run, substrate and product were extracted and the CLEC were washed twice with a solution of $\mathrm{CH}_{3} \mathrm{CN}: \mathrm{H}_{2} \mathrm{O}$. After the removal of the supernatant, a fresh solution of substrate and oxidant was added and a second catalytic run was performed. A drop from $86 \%$ to $20 \%$ of the sulfoxide yield was observed, attesting that the catalytic system is rather unstable under harsh oxidative conditions. To discriminate between the catalyst and the protein degradation, after the second run, a reloading step was done by soaking in situ the CLECNikA-FeL3 with a fresh FeL3 solution. A third catalytic run was then performed leading to a yield of $84 \%$ in $2 a$. The full restored activity cannot be attributed to the complex alone in solution (see Figure 2, FeL3 complex activity is only $3 \%$ ), suggesting that active CLEC were recovered and that the iron complex is degraded at first while the NikA protein scaffold seems less impacted. Nevertheless after a new consecutive run, as expected, the activity dropped again but could not be restored after CLEC soaking with FeL3. Therefore, the $\mathrm{NaOCl}$ is too harsh for the complex and the protein, leading to a moderate stability. The use of other oxidants such as hydrogen peroxide (even in the presence of acetic acid) ${ }^{[29]}$ or tert-butyl hydroperoxide, were not conclusive with both lower yields and slower kinetics. The results nevertheless displayed an activity higher than most of the other hybrid systems in solution reported in the literature, with a total of 470 TON obtained after the fourth run. It has to be mentioned that in similar conditions but in solution, a second run did not yield any product.

In addition, the CLEC strategy allowed us to expand the substrate scope to water-insoluble substrates (Table 1), using a $15 \mu \mathrm{M}$ of CLECNikA-FeL3 (50 CLEC) as catalyst for screening. It has to be noted that in these experimental conditions, 1a conversion after $2 \mathrm{~h}$ was less efficient (15\% instead of $84 \%$ ) as the catalyst concentration was half of the amount used above. This nearly six-fold reduced yield was used as reference for comparison. We observed that the CLECNikA-FeL3 is efficient for 1a-d oxidation with a high selectivity for sulfoxide formation, reproducing the trends observed in solution with the hybrid soNikA-FeL2 (1) still more reactive than 1a, 1c or 1d) ${ }^{[9]}$ suggesting an electrophilic character of the oxidizing species. This is also confirmed by the use of a 4-nitropenyl- $N$ phenylthioglycolamide derivative, which was reluctant to any transformation under oxidative conditions. The lower transformation of 1c and 1d, compared to soNikA-FeL2 in solution, ${ }^{[9]}$ strongly suggests that steric effects related to the methoxy groups are more important in the solid state since both substrates were totally soluble.

Thanks to the stability of the CLEC toward organic solvents, more lipophilic substrates 1e-1i were then targeted. This new catalytic system allows now to oxidize less enriched thioethers (and aliphatic) like 1e. Interestingly, the use of a $\mathrm{CH}_{3} \mathrm{CN}: \mathrm{H}_{2} \mathrm{O}$ allowed the solubilization of naphthyl moiety containing substrates $\mathbf{1 f - i}$. Comparing $\mathbf{1 f}$ to $\mathbf{1 a}$, the presence of a naphthyl versus a phenyl moiety strongly decreases the reactivity and steric effects can be evoked. This is also confirmed by comparing $\mathbf{1 b}$ and $\mathbf{1 g}$ with a yield divided by 2 after 2 hours. Nevertheless, $\mathbf{2} \mathbf{g}$ and $\mathbf{2} \mathbf{i}$ were obtained with an efficiency at the level of $\mathbf{2 a}$. The best yield is observed for $\mathbf{2 e}$, related again to steric effects. The best yields obtained for $\mathbf{2 b}$ and $\mathbf{2 e}$ then pushed us to test the catalytic system under a $31 \mu \mathrm{M}$ concentration for a direct comparison with the standard reaction conditions (Figure 2, Table S2). The results after two hours gave a $100 \%$ selectivity and a yield of $80 \%$ (204 TON) and $89 \%$ (227 TON) for $\mathbf{2 b}$ and $\mathbf{2 e}$, respectively, in the line with the results obtained for $\mathbf{2 a}$. The test the limits of the catalyst, $\mathbf{1 b}$ was oxidized with only $0.09 \%$ of catalyst, giving a slightly increased yield (50\%) of $\mathbf{2 b}$ but a 8 fold increased TON (570). To conclude, by switching from homogeneous to heterogeneous conditions, we have then afforded a larger promiscuity for substrates.

Finally, in addition to the role of CLEC on substrate promiscuity, the most important output of this study is the kinetics of the S1 oxidation. We estimated a 5-fold increase of the sulfoxide turnover frequency to $3.7 \mathrm{~min}^{-1}$ against $0.7 \mathrm{~min}^{-1}$ in solution under standard conditions. In addition, we measured the kinetics when $\mathrm{H}_{2} \mathrm{O}_{2}$ was used as the oxidant, taking advantage of the slower reactivity. We then measured a 3 fold increase $\left(0.34 \mathrm{~min}^{-1}\right.$ vs $0.12 \mathrm{~min}^{-1}$ Figure S4). This rate increase emphasizes the positive impact of the combination of a high catalyst density within the crystals and a better catalyst stability on the catalytic efficiency. 


\begin{tabular}{|c|c|c|c|c|}
\hline & $\begin{array}{c}\text { Product } \\
2 \mathrm{a}-2 \mathrm{i}\end{array}$ & CLECNikA-FeL3 & $\begin{array}{l}\text { Yield } \\
2 \mathrm{a}-2 \mathrm{i} \\
(\%) \\
\end{array}$ & TON \\
\hline \multicolumn{5}{|l|}{$2 a$} \\
\hline & & + & 15 & 38 \\
\hline & & $+*$ & 84 & 214 \\
\hline \multirow[t]{4}{*}{$2 \mathrm{~b}$} & & - & 0 & - \\
\hline & & + & 28 & 71 \\
\hline & & $+*$ & 80 & 202 \\
\hline & & $+* *$ & 50 & 570 \\
\hline \multirow[t]{2}{*}{$2 c$} & & - & 0 & - \\
\hline & & + & 1 & 2 \\
\hline \multirow[t]{2}{*}{ 2d } & & - & 3 & - \\
\hline & & + & 17 & 43 \\
\hline \multirow[t]{3}{*}{$2 e$} & & - & 2 & - \\
\hline & & + & 30 & 77 \\
\hline & & $+*$ & 89 & 227 \\
\hline \multirow[t]{2}{*}{$2 \mathrm{f}$} & & - & 4 & - \\
\hline & & + & 5 & - \\
\hline \multirow[t]{2}{*}{$2 \mathrm{~g}$} & & - & 5 & - \\
\hline & & + & 15 & 40 \\
\hline \multirow[t]{2}{*}{$2 \mathrm{~h}$} & & - & 0 & - \\
\hline & & + & 0 & - \\
\hline \multirow[t]{2}{*}{$2 \mathbf{i}$} & & - & 3 & - \\
\hline & & + & 15 & 38 \\
\hline
\end{tabular}

Experimental conditions: 1:255:600; [cat] = $15 \mu \mathrm{M}$ in in $\mathrm{CH}_{3} \mathrm{CN}: \mathrm{H}_{2} \mathrm{O}$ 1/1 v:v for 2 hours. ${ }^{*}$ With $31 \mu \mathrm{M}$ CLEC. ${ }^{* *}$ With 1:1150:1200.

Conclusion. This study shows that the transition from homogeneous to heterogeneous conditions does not affect the selectivity of the so/NikA-FeLn artificial enzymes, confirming that the switch of conditions consists of a real transposition of the catalysis. It also confirms that the reaction mechanism of the so/NikA-FeLn is conserved. The use of CLECNikA-FeLn has increased the reactivity by nearly $1 / 3^{\text {rd }}$ in one standard run of catalysis, 3.5 -fold when lowering the catalyst concentration to $0.09 \%$ and 3 -fold when reloading the catalyst. The moderate stability of the catalysts closely relates to the kinetic of the reaction, as the faster the oxidant reacts with the substrate, the less it degrades the catalyst, related to the nucleophilicity of the sulfide substrate. No positive results were conclusive for enantioselectivity implying that NikA engineering approaches have to be considered in the future. For industrial approaches, the CLEC technology should be developed for Modafinil® or Esomeprazole ${ }^{\circledR}$ transformation. Finally, this work illustrates the mode of action of artificial CLEC on substrate promiscuity and kinetics enhancement.

\section{Acknowledgements}


We thank the Agence Nationale pour la Recherche for grant (ANR-14-CE06-0005-01), and the CEA, CNRS and Univ. Grenoble-Alpes for their institutional support. This work has been partially supported by Labex ARCANE (ANR-11-LABEX-0003-1) and CBH-EUR-GS (ANR-17-EURE-0003). We thank the staffs from the BM-30A beamline of the European Synchrotron Facility in Grenoble

Keywords: Heterogeneous Catalysis • Oxidation • Artificial Metalloenzymes $•$ Sulfoxidation $\mathrm{k} \cdot$ Bioinspired catalyst $\bullet$ Crystal Catalysis

[1] a) P. T. Anastas, M. M. Kirchhoff, Acc. Chem. Res. 2002, 35, 686-694; b) M. Poliakoff, J. M. Fitzpatrick, T. R. Farren, P. T. Anastas, Science 2002, 297, 807-810; c) R. A. Sheldon, Chem. Soc. Rev. 2012, 41, 1437-1451; d) R. A. Sheldon, D. Brady, ChemSusChem 2019, 12, 2859-2881.

[2] a) M. Hönig, P. Sondermann, N. J. Turner, E. M. Carreira, Angew. Chem. Int. Ed. 2017, 56, 8942-8973; b) U. T. Bornscheuer, G. W. Huisman, R. J. Kazlauskas, S. Lutz, J. C. Moore, K. Robins, Nature 2012, 485, 185-194; c) M. T. Reetz, in Directed Evolution of Selective Enzymes, Wiley-VCH Verlag \& Co, 2016, pp. 237-266.

[3] a) G. Li, M. Garcia-Borràs, M. Fürst, A. Ilie, M. Fraaije, K. Houk, M. Reetz, J. Am. Chem. Soc. 2018, 140; b) M. T. Reetz, Acc. Chem. Res. 2019, 52, 336-344.

[4] Y. Yu, C. Cui, X. Liu, I. D. Petrik, J. Wang, Y. Lu, J. Am. Chem. Soc. 2015, 137, 11570-11573.

[5] M. E. Wilson, G. M. Whitesides, J. Am. Chem. Soc. 1978, 100, 306-307.

[6] a) F. Schwizer, Y. Okamoto, T. Heinisch, Y. Gu, M. M. Pellizzoni, V. Lebrun, R. Reuter, V. Köhler, J. C. Lewis, T. R. Ward, Chem Rev. 2018, 118, 142-231; b) F. Yu, V. M. Cangelosi, M. L. Zastrow, M. Tegoni, J. S. Plegaria, A. G. Tebo, C. S. Mocny, L. Ruckthong, H. Qayyum, V. L. Pecoraro, Chem. Rev. 2014, 114, 3495-3578; c) K. Chen, F. H. Arnold, Nature Catalysis 2020, 3, 203-213; d) P. N. Devine, R. M. Howard, R. Kumar, M. P. Thompson, M. D. Truppo, N. J. Turner, Nature Reviews Chemistry 2018, 2, 409-421; e) D. F. Qi, C. M. Tann, D. Haring, M. D. Distefano, Chem. Rev. 2001, 101, 3081-3111.

[7] a) H. Cotton, T. Elebring, M. Larsson, L. Li, H. Sörensen, S. von Unge, Tetrahedron: Asymmetry 2000, 11, 3819-3825; b) L. Olbe, E. Carlsson, P. Lindberg, Nature Reviews Drug Discovery 2003, 2, 132-139; c) H.-J. Federsel, Chirality 2003, 15, S128-S142; d) J. Legros, J. R. Dehli, C. Bolm, Adv. Synth. Cat. 2005, 347, 19-31; e) E. Wojaczynska, J. Wojaczynski, Chem. Rev. 2010, 110, 43034356; f) J. W. Young, K. Kooistra, M. A. Geyer, Neuropsychopharmacology 2011, 36, 1385-1396; g) K. A. Stingl, K. M. Weiß, S. B. Tsogoeva, Tetrahedron 2012, 68, 8493-8501.

[8] S. Wu, R. Snajdrova, J. C. Moore, K. Baldenius, U. T. Bornscheuer, Angew. Chem. Int. Ed., 2020,59,2-34.

[9] C. Esmieu, M. V. Cherrier, P. Amara, E. Girgenti, C. Marchi-Delapierre, F. Oddon, M. lannello, A. Jorge-Robin, C. Cavazza, S. Ménage, Angew. Chem. Int. Ed. 2013, 52, 3922-3925.

[10] a) T. Komatsu, S. Ishihara, E. Tsuchida, H. Nishide, C. Morokuma, S. Nakamura, Biomacromolecules 2005, 6, 1489-1494; b) R Ricoux, M. Allard, R. Dubuc, C. Dupont, J. D. Marechal, J. P. Mahy, Org. Biomol. Chem. 2009, 7, 3208-3211; c) M. Allard, C. Dupont, V. M. Robles, N. Doucet, A. Lledos, J. D. Marechal, A. Urvoas, J. P. Mahy, R. Ricoux, Chembiochem 2012, 13, $240-251$.

[11] a) A. Mahammed, Z. Gross, J. Am. Chem. Soc. 2005, 127, 2883-2887; b) P. Rousselot-Pailley, C. Bochot, C. Marchi-Delapierre, A Jorge-Robin, L. Martin, J. C. Fontecilla-Camps, C. Cavazza, S. Menage, Chembiochem 2009, 10, 545-552; c) J. Tang, F. Huang, Y. Wei, H. Bian, W. Zhang, H. Liang, Dalton Trans. 2016, 45, 8061-8072.

[12] F. van de Velde, L. Konemann, F. van Rantwijk, R. A. Sheldon, Chem. Commun. 1998, 1891-1892.

[13] T. K. Hyster, L. Knorr, T. R. Ward, T. Rovis, Science 2012, 338, 500-503.

[14] L. Rondot, E. Girgenti, F. Oddon, C. Marchi-Delapierre, A. Jorge-Robin, S. Ménage, Journal of Molecular Catalysis A: Chemical 2016, $416,20-28$.

[15] a) E. Sansiaume-Dagousset, A. Urvoas, K. Chelly, W. Ghattas, J.-D. Maréchal, J.-P. Mahy, R. Ricoux, Dalton Trans. 2014, 43, 83448354; b) R. Ricoux, M. Allard, R. Dubuc, C. Dupont, J.-D. Maréchal, J.-P. Mahy, Org. Biomol. Chem. 2009, 7, 3208-3211; c) S. Nimri, E. Keinan, J. Am. Chem. Soc. 1999, 121, 8978-8982.

[16] M. Pott, T. Hayashi, T. Mori, P. R. E. Mittl, A. P. Green, D. Hilvert, J. Am. Chem. Soc. 2018, 140, 1535-1543.

[17] A. Pordea, M. Creus, J. Panek, C. Duboc, D. Mathis, M. Novic, T. R. Ward, J. Am. Chem. Soc. 2008, 130, 8085-8088.

[18] a) D. K. Garner, L. Liang, D. A. Barrios, J.-L. Zhang, Y. Lu, ACS Catal. 2011, 1, 1083-1089; b) C. Buron, K. Sénéchal-David, R. Ricoux, J.-P. Le Caër, V. Guérineau, P. Méjanelle, R. Guillot, C. Herrero, J.-P. Mahy, F. Banse, Chem. Eur. J. 2015, 21, 1218812193; c) J. R. Carey, S. K. Ma, T. D. Pfister, D. K. Garner, H. K. Kim, J. A. Abramite, Z. Wang, Z. Guo, Y. Lu, J. Am. Chem. Soc. 2004, 126, 10812-10813.

[19] L. Leone, D. D'Alonzo, V. Balland, G. Zambrano, M. Chino, F. Nastri, O. Maglio, V. Pavone, A. Lombardi, Frontiers in Chemistry 2018, 6 .

[20] a) D. N. Tran, K. J. Balkus, ACS Catal. 2011, 1, 956-968; b) R. A. Sheldon, S. van Pelt, Chem. Soc. Rev. 2013, 42, 6223-6235; c) C. Garcia-Galan, Á. Berenguer-Murcia, R. Fernandez-Lafuente, R. C. Rodrigues, Adv. Synth. Cat. 2011, 353, 2885-2904; d) R. Chapman, M. H. Stenzel, J. Am. Chem. Soc. 2019, 141, 2754-2769.

[21] M. V. Cherrier, E. Girgenti, P. Amara, M. Iannello, C. Marchi-Delapierre, J. C. Fontecilla-Camps, S. Menage, C. Cavazza, J. Biol. Inorg. Chem. 2012, 17, 817-829.

[22] a) S. Wu, R. Snajdrova, J. C. Moore, K. Baldenius, U. T. Bornscheuer, Angew. Chem. Int. Ed. 2020, 59, 2-34; b) A. Schmid, J. S. Dordick, B. Hauer, A. Kiener, M. Wubbolts, B. Witholt, Nature 2001, 409, 258-268.

[23] a) R. A. Persichetti, N. L. S. Clair, J. P. Griffith, M. A. Navia, A. L. Margolin, J. Am. Chem. Soc. 1995, 117, 2732-2737; b) A. L. Margolin, M. A. Navia, Angew. Chem. Int. Ed. 2001, 40, 2204-2222; c) F. A. Quiocho, F. M. Richards, PNAS 1964, 52, 833-839; d) I. Luiz de Mattos, L. V. Lukachova, L. Gorton, T. Laurell, A. A. Karyakin, Talanta 2001, 54, 963-974; e) J. J. Roy, E. T. Abraham, Chem. Rev. 2004, 104, 3705-3722.

[24] a) H. Tabe, S. Abe, T. Hikage, S. Kitagawa, T. Ueno, Chem. Asian J. 2014, 9, 1373-1378; b) S. Abe, B. Maity, T. Ueno, Chem. Commun. 2016, 52, 6496-6512.

[25] M. Costas, A. K. Tipton, K. Chen, D. H. Jo, L. Que, J. Am. Chem. Soc. 2001, 123, 6722-6723.

[26] P. Mialane, A. Nivorojkine, G. Pratviel, L. Azéma, M. Slany, F. Godde, A. Simaan, F. Banse, T. Kargar-Grisel, G. Bouchoux, J. Sainton, O. Horner, J. Guilhem, L. Tchertanova, B. Meunier, J.-J. Girerd, Inorg. Chem. 1999, 38, 1085-1092.

[27] J. I. Elliott, J. M. Brewer, Arch. Biochem. Biophys. 1979, 192, 203-213.

[28] J. Tang, P. Yao, F. Huang, M. Luo, Y. Wei, H. Bian, Tetrahedron: Asymmetry 2017, 28, 1700-1707.

[29] R. Mas-Balleste, L. Que, J. Am. Chem. Soc. 2007, 129, 15964-15972. 


\section{Entry for the Table of Contents}

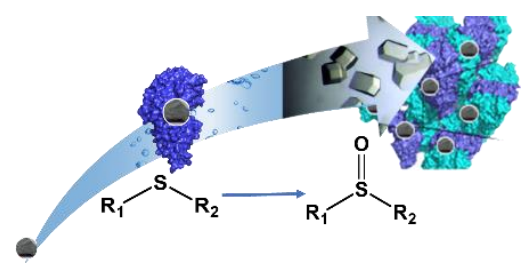

Crystals are not fragile. Using protein crystals as supports for inorganic catalyst, we have designed a methodology of heterogeneous catalysis by artificial enzymes. The hybrid solids, made from the association of the NikA protein and an iron complex with a N2Py2 ligand, provide access to a larger substrate scope, a greater stability and greater kinetics for sulfide transformations than the homogeneous counterpart. 